TRANSACTIONS OF THE

AMERICAN MATHEMATICAL SOCIETY

Volume 185, November 1973

\title{
EXTREME POINTS FOR SOME CLASSES OF UNIVALENT FUNCTIONS (1)
}

\author{
BY

\section{W. HENGARTNER AND G. SCHOBER}

\begin{abstract}
Monotonicity properties are given for extreme points in classes of normalized analytic and univalent mappings of an arbitrary domain. For the familiar class of normalized univalent mappings of the unit disk, extreme points $f$ are shown to have the remarkable property that $f / z$ is univalent.
\end{abstract}

Introduction. Let $D$ be an arbitrary domain in the complex plane and $H(D)$ the set of all analytic functions in $D$. For a subset $\Im$ of $H(D)$ we denote by $E_{9}$ the set of extreme points of $\Im$; i.e., each $f \in E_{\Im}$ is not a proper convex combination of other elements of $\exists$. With the topology of uniform convergence on compact subsets, $H(D)$ becomes a locally convex linear topological space. If $\ni$ is a compact subset of $H(D)$, it is well known that $E_{9}$ is nonempty and that for each continuous linear functional $L$ on $H(D)$, there exists an $f \in E_{\rho}$ such that

$$
\operatorname{Re} L(f)=\max _{g \in 9} \operatorname{Re} L(g) \text {. }
$$

That is, linear extremal problems may be solved just by examining extreme points.

We shall be concerned with properties of extreme points for three classes of functions:

$$
\begin{aligned}
& \mathcal{Y}_{1}=\left\{f \in H(D): f \text { is univalent, } f(p)=P \text {, and } f^{\prime}(p)=\mathfrak{p}\right\}, \\
& \mathcal{Y}_{2}=\{f \in H(D): f \text { is univalent, } f(p)=P \text {, and } f(q)=Q\},
\end{aligned}
$$

where $p, q \in D$ and $\mathfrak{p}, P, Q \in \mathbf{C}(\mathfrak{p} \neq 0)$ are fixed, and the customary normalized schlicht class in the unit disk $U=\{|z|<1\}$,

$$
S=\left\{f \in H(U): f \text { is univalent, } f(0)=0 \text {, and } f^{\prime}(0)=1\right\} \text {. }
$$

The familiar normalizations for these classes insure that they are compact.

We shall show that extreme points for $\vartheta_{1}$ and $\vartheta_{2}$ have strong geometric properties. They map onto slit domains for which each component of the complement is a monotone arc. In the case of $\Im_{1}$ the monotonicity is with respect to the family of circles with center $P$. In the case of $\mathcal{Y}_{2}$ the monotonicity is with respect to the family of ellipses with foci $P$ and $Q$. In a sense, the ellipse family for $\mathcal{Y}_{2}$ is the natural extension of the circle family for $\mathcal{Y}_{1}$.

Received by the editors March 24, 1972 and, in revised form, July 12, 1972.

AMS (MOS) subject classifications (1970). Primary 30A36; Secondary 30A40.

Key words and phrases. Univalent functions, extreme points

(1) This research was supported in part by the National Research Council of Canada Grant A-7339 and National Science Foundation Grant GP-19694. 
Since $S$ is a special case of $\mathcal{Y}_{1}$, an extreme point of $S$ maps onto the complement of an arc which goes monotonically to $\infty$ (cf. also [1]). We shall show that such mappings have the remarkable property that $f / z$ is also univalent. In addition, we obtain some geometric properties of $f / z$.

The class $\vartheta_{1}$. The following theorem is due to L. Brickman [1] in case $D$ is the unit disk. It is worth emphasizing that his method carries over to arbitrary domains $D$. We include a proof for completeness.

Theorem 1. Let $f \in E_{\Im_{1}}$. Then $\overline{f(D)}=\mathrm{C}$, and each component of $\mathbf{C}-f(D)$ is either $a$ point or a Jordan arc which meets each circle with center $P$ in at most one point. Only one component is unbounded.

Proof. Let $f \in \mathcal{Y}_{1}$, and suppose that $a$ and $b$ are distinct points belonging to a component of $\mathbf{C}^{*}-f(D)$. Here $\mathbf{C}^{*}$ is the extended plane $\mathbf{C} \cup\{\infty\}$. Then one may choose a single valued branch of $\sqrt{(w-a)(w-b)}=\psi(w)$ in $f(D)$, and the functions

$$
\begin{aligned}
& \varphi_{1}(w)=\frac{w+\psi(w)-\psi(P)+P \psi^{\prime}(P)}{1+\psi^{\prime}(P)}, \\
& \varphi_{2}(w)=\frac{w-\psi(w)+\psi(P)-P \psi^{\prime}(P)}{1-\psi^{\prime}(P)}
\end{aligned}
$$

are univalent in $f(D)$. Furthermore, $\varphi_{1} \circ f, \varphi_{2} \circ f \in \mathcal{Y}_{1}$, and one has

$$
f=\lambda \varphi_{1} \circ f+(1-\lambda) \varphi_{2} \circ f
$$

for $\lambda=\frac{1}{2}\left[1+\psi^{\prime}(P)\right]$. Consequently, $f$ cannot be an extreme point of $\mathcal{Y}_{1}$ if $\lambda \in(0,1)$, or if

$$
\psi^{\prime}(P) \in(-1,1) .
$$

In case $a=P+r e^{i a}$ and $b=P+r e^{i \beta}$, then

$$
\left[\psi^{\prime}(P)\right]^{2}=\cos ^{2} \frac{1}{2}(\alpha-\beta) \in[0,1) .
$$

Therefore $f$ cannot be an extreme point if any component of $C^{*}-f(D)$ meets any circle about $P$ in more than one point. For $f \in E_{\Omega_{1}}$ it follows immediately (e.g., as in [1]) that each component of $\mathrm{C}-f(D)$ is a point or a Jordan arc and hence $\overline{f(D)}=\mathrm{C}$. Two components cannot be unbounded, for otherwise they would belong to a single component of $C^{*}-f(D)$ in which there would be distinct points on all sufficiently large circles about $P$.

Remark. The same conclusion and proof hold in case the normalization for the class $\Im_{1}$ is altered so that $f^{\prime}(p)$ belongs to a fixed set $A \subset \mathbf{C}\left(\right.$ e.g., $m \leq\left|f^{\prime}(p)\right|$ $\leq M$ for some $0<m \leq M<\infty)$. Of course, $\Im_{1}$ will be compact iff $A$ is compact.

The class $\mathcal{F}_{2}$. Theorem 1 extends in a geometrically pleasing way to the class $\Im_{2}$. 
Theorem 2. Let $f \in E_{9_{2}}$. Then $\overline{f(D)}=\mathrm{C}$, and each component of $\mathbf{C}-f(D)$ is either a point or a Jordan arc which meets each ellipse with foci $P$ and $Q$ in at most one point. Only one component is unbounded.

Proof. Let $f \in \mathcal{Y}_{2}$, and suppose that $a$ and $b$ are distinct points belonging to a component of $\mathbf{C}^{*}-f(D)$. Then $\psi(w)=\sqrt{(w-a)(w-b)}$ has a single valued branch in $f(D)$, and the functions

$$
\begin{aligned}
& \phi_{1}(w)=\frac{(P-Q)[w+\psi(w)]+Q \psi(P)-P \psi(Q)}{P-Q+\psi(P)-\psi(Q)}, \\
& \phi_{2}(w)=\frac{(P-Q)[w-\psi(w)]-Q \psi(P)+P \psi(Q)}{P-Q-\psi(P)+\psi(Q)}
\end{aligned}
$$

are univalent in $f(D)$. Furthermore, $\phi_{1} \circ f, \phi_{2} \circ f \in \mathcal{I}_{2}$, and one has

$$
f=\lambda \phi_{1} \circ f+(1-\lambda) \phi_{2} \circ f
$$

for

$$
\lambda=\frac{1}{2}+\frac{1}{2}(\psi(P)-\psi(Q)) /(P-Q) .
$$

Consequently, $f$ cannot be an extreme point if $\lambda \in(0,1)$, or if

$$
(\psi(P)-\psi(Q)) /(P-Q) \in(-1,1) .
$$

If $a$ and $b$ lie on an ellipse with foci $P$ and $Q$, then

$$
\begin{aligned}
& a=\frac{1}{2}(P+Q)+\frac{1}{2}(P-Q) h(\alpha) \text { and } \\
& b=\frac{1}{2}(P+Q)+\frac{1}{2}(P-Q) h(\beta)
\end{aligned}
$$

where $h(\theta)=\varepsilon^{-1} \cos \theta+i \varepsilon^{-1} \sqrt{1-\varepsilon^{2}} \sin \theta$ and $\varepsilon$ is the eccentricity of the ellipse. Writing $h(\theta)=\cos (\theta-i \eta)$ where $\cosh \eta=\varepsilon^{-1}$, we find

$$
\begin{aligned}
{[h(\alpha) h(\beta)-\cos (\alpha-\beta)]^{2} } & =\sin ^{2}(\alpha-i \eta) \sin ^{2}(\beta-i \eta) \\
& =\left[1-h^{2}(\alpha)\right]\left[1-h^{2}(\beta)\right] .
\end{aligned}
$$

In terms of $\psi,(16)$ becomes

$$
\left[\frac{\psi^{2}(P)+\psi^{2}(Q)}{(P-Q)^{2}}-\cos ^{2}\left(\frac{\alpha-\beta}{2}\right)\right]^{2}=\frac{4 \psi^{2}(P) \psi^{2}(Q)}{(P-Q)^{4}} .
$$

So

$$
\frac{\psi^{2}(P)+\psi^{2}(Q)}{(P-Q)^{2}}-\cos ^{2}\left(\frac{\alpha-\beta}{2}\right)= \pm \frac{2 \psi(P) \psi(Q)}{(P-Q)^{2}}
$$

where we must determine the sign of the right side correctly. This may be done geometrically, or by a continuity argument we may determine the sign by checking when $b$ is opposite $a$. Indeed, $\psi(P)$ and $\psi(Q)$ may be defined as continuous functions of $b$ as $b$ moves along the ellipse to the point $b=$ 
$P+Q-a$ opposite $a$, and for this point $\psi(P)=\psi(Q)$ and the cosine term vanishes, so that the + sign is correct in (18). Therefore

$$
\left[\frac{\psi(P)-\psi(Q)}{P-Q}\right]^{2}=\cos ^{2}\left(\frac{\alpha-\beta}{2}\right)
$$

and (14) is satisfied. Consequently, $f$ cannot be an extreme point if any component of $C^{*}-f(D)$ meets an ellipse with foci $P$ and $Q$ in more than one point. The remaining assertions follow easily as in Theorem 1.

Remark 1. The same conclusion and proof are valid in case the normalization for $\mathcal{Y}_{2}$ is relaxed so that $q \in \bar{D}$.

Remark 2. Note that Theorem 2 is a natural extension of Theorem 1. For if $Q \rightarrow P$, then the analytic condition (14) becomes the corresponding one (8) in Theorem 1. Geometrically, the ellipses degenerate into circles as the family $\mathcal{Y}_{2}$ is deformed into $\Im_{1}$.

The class $S$. Let $f$ be an extreme point of $S$. The principal content of the following theorem is that $f / z$ is univalent. This is obviously false for $S$ in general (e.g. the identity function). It is a rather remarkable property of the extreme points.

Theorem 3. Let $f \in E_{s}$. Then

(i) $\log (f / z)$ is univalent in the unit disk $U$.

(ii) $\log (f / z)$ maps $U$ onto a domain $\Omega$ convex in the $v$-direction, i.e., the intersection of $\Omega$ with each vertical line is connected.

(iii) The vertical line with abcissa $x$ meets $\bar{\Omega}$ in a segment of length $l_{x}<2 \pi$.

(iv) $l_{x}$ is a continuous strictly increasing function of $x$ on $\left[x_{0}, \infty\right), \lim _{x \rightarrow \infty} l_{x}=2 \pi$, and $l_{x_{0}}=0$ where $x_{0}=\inf \{\operatorname{Re} z: z \in \Omega\}$.

(v) $\mathrm{f} / \mathrm{z}$ is univalent in $U$.

(vi) $f / z$ maps $U$ onto a domain $\Omega_{1}$ with the property that each circle $|w|=r$ meets $\bar{\Omega}_{1}$ in a single arc of $\omega_{r}$ radians. Moreover, $\omega_{r}$ is a continuous strictly increasing function of $r$ on $\left[r_{0}, \infty\right), \lim _{r \rightarrow \infty} \omega_{r}=2 \pi$, and $\omega_{r_{0}}=0$ where $r_{0}=\inf \left\{|w|: w \in \Omega_{1}\right\}$.

Proof. Suppose $f \in E_{S}$. By Theorem $1, \mathrm{C}-f(U)$ is a Jordan arc extending monotonically from a finite point $P_{2}$ to $\infty$. So the restriction of $f(U)$ to the disk $|w|<n$ is a domain $D_{n}$. Let $f_{n}$ map $U$ conformally onto $D_{n}$ with $f_{n}(0)=0$ and $f_{n}^{\prime}(0)>0$. Then by the convergence theorem of Radó [3], $f_{n}$ converges to $f$ uniformly on $U$ relative to the spherical metric. Suppose $f\left(e^{i \theta_{2}}\right)=f_{n}\left(e^{i \theta_{2 n}}\right)=P_{2}$ and $f\left(e^{i \theta_{1}}\right)=\infty,\left|f_{n}\left(e^{i \theta_{1, n}}\right)\right|=n$, where we choose $0<\theta_{2}-\theta_{1}<2 \pi$. Of course, the choice of $e^{i \theta_{1, n}}$ is not unique. Nevertheless, $e^{i \theta_{1, n}} \rightarrow e^{i \theta_{1}}$ and $e^{i \theta_{2 n}} \rightarrow e^{i \theta_{2}}$ as $n \rightarrow \infty$ by the uniform convergence. Let $\Xi_{n}$ be the Möbius self-mapping of $U$ with $\mathfrak{S}_{n}(1)=e^{i \theta_{1 n}}, \quad \mathfrak{S}_{n}(-1)=e^{i \theta_{2 n}}$, and $\mathfrak{S}_{n}(i)=e^{(1 / 2) i\left(\theta_{1 n}+\theta_{2 n}\right)}$, and denote $s$ $=\lim _{n \rightarrow \infty} \mathfrak{B}_{n}$.

If we choose the branch of the logarithm for which $\log 1=0$, we have for $G_{n}=\log \left[\left(f_{n} \circ \mathfrak{g}_{n}\right) / \mathfrak{g}_{n}\right]$ the Poisson representation 


$$
\begin{aligned}
G_{n}(\zeta) & =\frac{1}{2 \pi} \int_{0}^{2 \pi} \log \left|\frac{f_{n} \circ \Xi_{n}\left(e^{i t}\right)}{\Xi_{n}\left(e^{i t}\right)}\right| \frac{e^{i t}+\zeta}{e^{i t}-\zeta} d t+i \text { (const) } \\
& =\frac{1}{2 \pi} \int_{0}^{2 \pi} \log \left|f_{n} \circ \Xi_{n}\left(e^{i t}\right)\right| \frac{e^{i t}+\zeta}{e^{i t}-\zeta} d t+i \text { (const). }
\end{aligned}
$$

So

$$
\begin{aligned}
G_{n}^{\prime}(\zeta) & =\frac{1}{\pi} \int_{0}^{2 \pi} \log \left|f_{n} \circ s_{n}\left(e^{i t}\right)\right| \frac{e^{i t}}{\left(e^{i t}-\zeta\right)^{2}} d t \\
& =\frac{1}{\pi i(1-\zeta)} \int_{0}^{2 \pi} \log \left|f_{n} \circ s_{n}\left(e^{i t}\right)\right| d\left(\frac{e^{i t}-1}{e^{i t}-\zeta}\right) \\
& =\frac{i}{\pi(1-\zeta)} \int_{0}^{2 \pi} \frac{e^{i t}-1}{e^{i t}-\zeta} d \log \left|f_{n} \circ s_{n}\left(e^{i t}\right)\right| .
\end{aligned}
$$

Since $\log \left|f_{n} \circ \xi_{n}\left(e^{i t}\right)\right|$ is nonincreasing for $0<t<\pi$ and nondecreasing for $\pi<t<2 \pi$, it follows that

$$
\begin{aligned}
& \operatorname{Re}\left\{\left(1-\zeta^{2}\right) G_{n}^{\prime}(\zeta)\right\} \\
& \quad=\frac{-1+|\zeta|^{2}}{\pi} \int_{0}^{2 \pi} \frac{\sin t}{\left|e^{i t}-\zeta\right|^{2}} d \log \left|f_{n} \circ \bar{s}_{n}\left(e^{i t}\right)\right| \geq 0 .
\end{aligned}
$$

By passing to the limit we have

$$
\operatorname{Re}\left\{\left(1-\zeta^{2}\right) G^{\prime}(\zeta)\right\} \geq 0
$$

for $G=\log (f \circ g / z)$. We have shown in [2] that (21) characterizes a particular class of univalent mappings to domains convex in the $v$-direction. Consequently, $\log (f / z)=G \circ \mathfrak{z}^{-1}$ is univalent and maps $U$ onto a domain $\Omega$ convex in the $u$ direction, so that (i) and (ii) are verified.

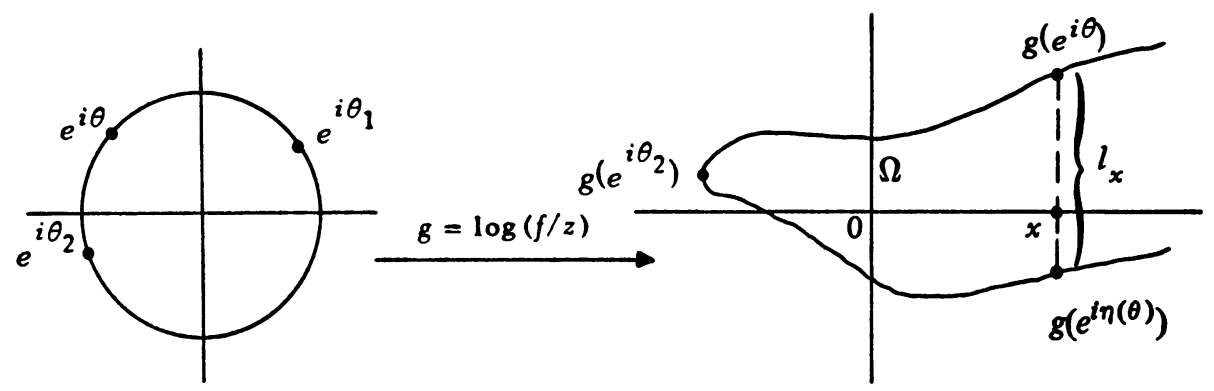

Figure 1

Since $\mathbf{C}-f(U)$ is a Jordan arc, we may define a decreasing function $\eta:\left[\theta_{1}, \theta_{2}\right] \rightarrow\left[\theta_{2}, \theta_{1}+2 \pi\right]$ such that 


$$
f\left(e^{i \eta(\theta)}\right)=f\left(e^{i \theta}\right) .
$$

Now for $g=\log (f / z)$ we have

$$
g\left(e^{i \theta}\right)-g\left(e^{i \eta(\theta)}\right)=i[\eta(\theta)-\theta] \quad \bmod 2 \pi i .
$$

Note that both sides of (23) are continuous functions of $\theta$ and vanish for $\theta=\theta_{2}$. We may therefore omit the mod $2 \pi i$. Since the right side of (23) is purely imaginary, necessarily

$$
g\left(e^{i \theta}\right)-g\left(e^{i \eta(\theta)}\right)=i l_{x}
$$

for $x=\operatorname{Re} g\left(e^{i \theta}\right)$ (see Figure 1). Therefore

$$
l_{x}=\eta(\theta)-\theta, \quad \theta \in\left(\theta_{1}, \theta_{2}\right] .
$$

For $\theta \in\left(\theta_{1}, \theta_{2}\right], \eta(\theta)-\theta$ is strictly decreasing. It is zero at $\theta_{2}$ and tends to $2 \pi$ at $\theta_{1}$. Since $x$ decreases as $\theta$ increases, the corresponding properties (iii) and (iv) of $l_{x}$ follow.

Since $l_{x}<2 \pi$ for all $x$, the exponential function is univalent in $\Omega$. Consequently $f / z=e^{g}$ is univalent, and (v) is proved. The properties described in (vi) then follow immediately from (iii) and (iv).

Remarks. (1) Since $f / z \neq 0$, univalent branches of $(f / z)^{1 / p}$ can be defined for $f \in E_{S}$ for all $p \geq 1$.

(2) Property (ii) and, in particular, (21) permit an analytic representation for $f \in E_{S}$ in terms of analytic functions with positive real part and the points $e^{i \theta_{1}}, e^{i \theta_{2}}$. One easily obtains estimates of various kinds from such representations. However, to obtain good results in this direction, one would also have to take into account the properties (iii) and (iv) of $l_{x}$.

(3) If $f(z)=\sum_{n=1}^{\infty} a_{n} z^{n} \in E_{S}$, then property (v) implies that

$$
\tilde{f}=a_{2}^{-1}[(f / z)-1] \in S .
$$

Since the $n$th coefficient of $\tilde{f}$ is $a_{n+1} / a_{2}$, elementary induction arguments become possible for estimating many linear functionals. However, one cannot expect very good results since $\tilde{f}$ is not a slit mapping. Nevertheless, several other approaches need to be explored which exploit the simultaneous univalence of $f$ and $\tilde{f}$.

\section{REFERENCES}

1. L. Brickman, Extreme points of the set of univalent functions, Bull. Amer. Math. Soc. 76 (1970), 372-374. MR 41 \#448.

2. W. Hengartner and G. Schober, On schlicht mappings to domains convex in one direction, Comment. Math. Helv. 45 (1970), 303-314. MR 43 \#3436.

3. T. Radó, Sur la représentation conforme des domaines variables, Acta. Sci. Math. (Szeged) 1 (1923), 180-186.

Department of Mathematics, Universite laval, Quebec, Qúbec, Canada

Department of Mathematics, Indiana University, Bloomington, Indiana 47401 